\title{
Limite supraforestière et changements environnementaux : pour une approche pluriscalaire et spatialisée des écosystèmes d'altitude
}

\author{
Timberline and environmental change : The importance of \\ scale and space for the study of high altitude ecosystems. \\ Baumgrenze und Umweltveränderungen: die Wichtigketi von \\ Maßstab und Raum beim Studium der Ökosysteme in großer \\ Höhe.
}

\section{Lydie Didier et Jean-Jacques Brun}

Volume 52, numéro 2, 1998

URI : https://id.erudit.org/iderudit/004786ar

DOI : https://doi.org/10.7202/004786ar

\section{Aller au sommaire du numéro}

\section{Éditeur(s)}

Les Presses de l'Université de Montréal

ISSN

0705-7199 (imprimé)

1492-143X (numérique)

Découvrir la revue

Citer cet article

Didier, L. \& Brun, J.-J. (1998). Limite supraforestière et changements environnementaux : pour une approche pluriscalaire et spatialisée des écosystèmes d'altitude. Géographie physique et Quaternaire, 52(2), 245-254. https://doi.org/10.7202/004786ar

\section{Résumé de l'article}

L'étude des successions post- pastorales dans l'étage subalpin en moyenne Maurienne (Savoie, France) amène les auteurs à une mise au point méthodologique et à une critique sur l'utilisation de la limite supraforestière comme indicateur des changements climatiques. Ils proposent une démarche hiérarchique et systémique pour aborder la complexité et l'hétérogénéité du phénomène « limite supraforestière ». Cette dernière est considérée comme une zone écotonale en mosaïque dont l'évolution peut être appréhendée (1) par rapport à une limite moyenne en observant cette mosaïque de loin, (2) en considérant les contacts entre les unités à la fois physionomiques et géomorphologiques qui constituent la mosaïque, ou (3) à l'échelle de lisières forestières individuelles. Les différentes trajectoires dynamiques recensées montrent que la place des lisières forestières au sein de la mosaïque phyto-morphologique est déterminante pour leurs potentialités d'évolution. La géomorphologie apparaît comme un facteur structurant fondamental des paysages et des dynamiques ligneuses. L'utilisation de la limite supraforestière comme indicateur des changements climatiques n'est envisageable que sous deux conditions : il faut décliner les paramètres climatiques à plusieurs échelles, en établissant une correspondance avec les processus dynamiques eux-mêmes pluriscalaires et il faut tenir compte du rôle du substrat, facteur fondamentalement spatial.
Tous droits réservés @ Les Presses de l'Université de Montréal,1998
Ce document est protégé par la loi sur le droit d'auteur. L’utilisation des services d'Érudit (y compris la reproduction) est assujettie à sa politique d'utilisation que vous pouvez consulter en ligne.

https://apropos.erudit.org/fr/usagers/politique-dutilisation/ 
LIMITE SUPRAFORESTIÈRE ET CHANGEMENTS ENVIRONNEMENTAUX : POUR UNE APPROCHE PLURISCALAIRE ET SPATIALISÉE DES ÉCOSYSTĖMES D'ALTITUDE

\begin{abstract}
Lydie DIDIER, Centre de Biologie alpine, Université Joseph Fourier, B.P. 53X, F-38041, Grenoble, Cedex 9, France. Jean-Jacques BRUN, Cemagref, division Environnement, Paysage et Forêt, B.P. 7688, F-38402, Saint-Martin-d'Hères, Cedex, France, jean-jacques.brun@cemagref.fr.
\end{abstract}

Manuscrit reçu le 19 septembre 1996 ; manuscrit révisé et accepté le 2 juin 1997

RÉSUMÉ L'étude des successions postpastorales dans l'étage subalpin en moyenne Maurienne (Savoie, France) amène les auteurs à une mise au point méthodologique et à une critique sur l'utilisation de la limite supraforestière comme indicateur des changements climatiques. Ils proposent une démarche hiérarchique et systémique pour aborder la complexité et l'hétérogénéité du phénomène «limite supraforestière ". Cette dernière est considérée comme une zone écotonale en mosaïque dont l'évolution peut être appréhendée (1) par rapport à une limite moyenne en observant cette mosaïque de loin, (2) en considérant les contacts entre les unités à la fois physionomiques et géomorphologiques qui constituent la mosaïque, ou (3) à l'échelle de lisières forestières individuelles. Les différentes trajectoires dynamiques recensées montrent que la place des lisières forestières au sein de la mosaïque phyto-morphologique est déterminante pour leurs potentialités d'évolution. La géomorphologie apparaît comme un facteur structurant fondamental des paysages et des dynamiques ligneuses. L'utilisation de la limite supraforestière comme indicateur des changements climatiques n'est envisageable que sous deux conditions : il faut décliner les paramètres climatiques à plusieurs échelles, en établissant une correspondance avec les processus dynamiques eux-mêmes pluriscalaires et il faut tenir compte du rôle du substrat, facteur fondamentalement spatial.
ABSTRACT Timberline and environmental change: The importance of scale and space for the study of high altitude ecosystems. The usefulness of the upper timberline as an indicator of climate change is reviewed in the light of the results of a study of subalpine post-pastoral succession in the Maurienne Valley (Savoie, France). The authors propose a hierarchical and systemic approach to take into account the complexity and heterogeneity of the timberline. The latter is considered as a mosaic ecotone whose evolution may be studied (1) by considering a mean line when observing the mosaic from far away, (2) by considering the boundaries between the physionomical and geomorphological units of the mosaic and (3) by focusing on the individual forest borders. The dynamic potentialities of the forest borders mainly depend on their place in the phytomorphological mosaic. Thus geomorphology is an important feature determining the landscape patterns and the recolonization patterns. The use of the timberline as an indicator of climate change is subjected to two conditions. Firstly, the climate parameters must be considered on a number of scales, according to the scales on which the vegetation dynamics itself may be studied. Secondly, the influence of the geomorphological parameters must not be neglected.
ZUSAMMENFASSUNG Baumgrenze und Umweltveränderungen : die Wichtigketi von Maßstab und Raum beim Studium der Ökosysteme in großer Höhe. Die Untersuchung der post-pastoralen Aufeinanderfolgen in der subalpinen Etage im mittleren Maurienne (Savoie, Frankreich) führt die Autoren zu einer methodologischen Korrektur und einer Kritik am der Benutzung der Baumgrenze als Indikator für Klimaveränderungen. Sie schlagen ein hierarchisches und systemkonformes Vorgehen vor, um die Komplexität und Heterogenität des Phänomens «Baumgrenze » anzugehen. Letztere wird als eine Übergangszone in Mosaikform angesehen, und man kann deren Entwicklung verstehen (1) in Bezug auf eine mittlere Grenze, wenn man das Mosaik von weitem beobachtet, (2) indem man die Berührungspunkte zwischen den physiognomischen und geomorphologischen Einheiten aus denen das Mosaik besteht, berücksichtigt, oder (3) indem man die individuellen Baumgrenzen studiert. Die verschiedenen erfassten dynamischen Entwicklungen zeigen, dass für das Entwicklungspotential der Waldränder ihr Platz innerhalb des phyto-morphologischen Mosaiks entscheidend ist. Die Geomorphologie bestimmt als ein fundamentaler strukturierender Faktor die Landschaften und die Baumdynamik. Man kann die Baumgrenze nur unter zwei Bedingungen als Indikator für klimatische Veränderungen benutzen : man muss die klimatischen Parameter in mehreren Maßstäben anwenden und dabei eine Konkordanz herstellen mit den dynamischen Prozessen, auf die ebenfalls mehrere Maßstäbe angewendet werden; und man muss die Rolle des Substrats berücksichtigen, des fundamentalen räumlichen Faktors. 


\section{INTRODUCTION}

La limite supérieure de la forêt est un trait majeur de l'organisation et du fonctionnement des écosystèmes d'altitude. Dans les paysages non soumis à l'action humaine, son déterminisme est essentiellement climatique et son altitude peut être considérée comme un "biothermomètre ". Ainsi, elle est souvent évoquée comme indicateur dans l'étude du changement climatique global. Or, la réponse de la limite supraforestière au réchauffement climatique actuel n'est pas simple à prévoir, puisqu'il s'agit d'un écotone complexe et souvent fortement marqué par l'occupation humaine. Dans les montagnes européennes, tant le défrichement et l'exploitation forestière et pastorale millénaires, que les reboisements de protection du XIXe et du XXe siècles ont modifié considérablement le paysage forestier jusqu'aux plus hautes altitudes. L'exode rural généralisé de ce siècle et la déprise des zones les plus reculées et les plus difficiles à exploiter sont à l'origine d'un enfrichement qui risque de masquer entièrement la réponse des écosytèmes au changement climatique. Ce reboisement spontané constitue cependant un excellent champ « expérimental » pour étudier les potentialités d'évolution des milieux d'altitude.

Dans la vallée de l'Arc, et surtout en basse et moyenne Maurienne (Savoie, Alpes françaises), les mutations socioéconomiques, ayant affecté les sociétés traditionnelles à partir du milieu du XIX ${ }^{e}$ siècle, se sont accompagnées de changements particulièrement profonds dans le paysage végétal. En effet, la réaction des écosystèmes de montagne à l'abandon des activités agricoles et pastorales a été rapide. En un demi siècle, elle a abouti à la formation de peuplements spontanés denses et sur des surfaces parfois considérables (Bozon et Grossi, 1991 ; Delcros, 1993 ; Brun et al., 1995 ; Grossi et al., 1995). En limite supérieure de forêt, la réponse des phytocénoses aux changements d'usage est moins bien étudiée. Elle semble moins spectaculaire, mais très diversifiée (Didier et Brun, 1997a). Les modalités de la recolonisation post-pastorale révèlent, en effet, l'hétérogénéité importante du milieu à l'étage subalpin. Ainsi, l'étude des phases récentes de colonisation ligneuse dans les alpages abandonnés de moyenne Maurienne (fig. 1) met en évidence plusieurs trajectoires d'évolution (Didier et Brun, 1995), permettant de mieux appréhender la réponse des phytocénoses dans le cas d'un réchauffement climatique. Ces recherches nous ont amené à une mise au point méthodologique et à une critique sur l'utilisation de la limite supraforestière comme indicateur des changements climatiques.

Avant de présenter les recherches menées en Maurienne, nous allons donner un bref aperçu de l'état actuel des connaissances sur la relation entre la limite supraforestière et le climat.
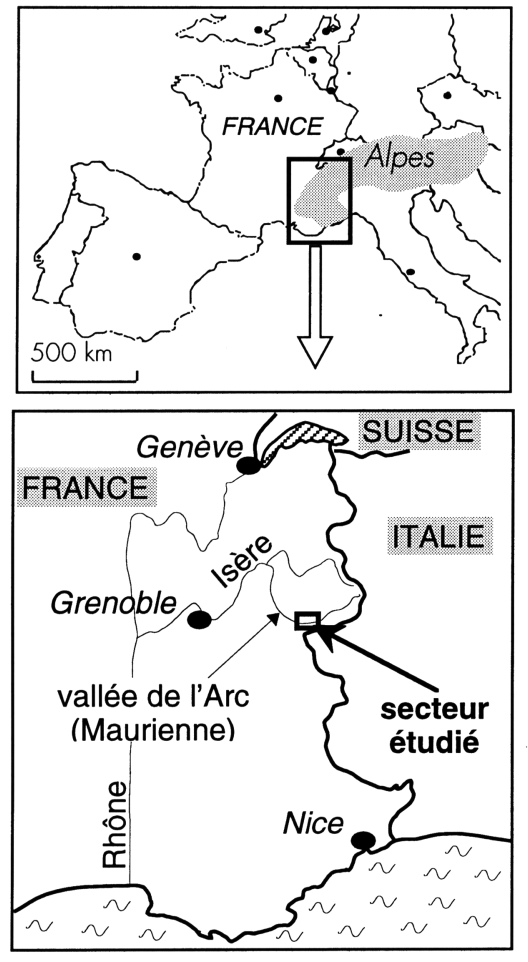

FIGURE 1. Localisation du secteur d'étude dans les Alpes françaises du Nord.

The study site in the northern French Alps.

\section{LA LIMITE SUPRAFORESTIÈRE : DÉTERMINISME, FLUCTUATIONS ET RÔLE DE BIO-INDICATEUR}

Les études sur la cause de l'absence de toute végétation arborée au-dessus d'une certaine limite altitudinale ont une longue tradition. Les synthèses sur les interactions multiples entre facteurs biotiques et facteurs abiotiques sont nombreuses (Troll, 1973 ; Tranquillini, 1979 ; Arno et Hammerly, 1984 ; Holtmeier, 1989, Slayter et Noble, 1992, par exemple). Théoriquement, les limites de répartition de toute espèce surviennent quand les individus ne peuvent plus maintenir un bilan positif de carbone à une étape donnée de leur cycle de vie (Slayter et Noble, 1992). D'après Tranquillini (1979), c'est la production limitée de matière sèche, combinée à la maturation incomplète des tissus et à une résistance insuffisante aux conditions climatiques, qui fait en sorte que les arbres ne peuvent plus résister à la dessiccation hivernale et sont éliminés au-dessus d'une certaine altitude. II est généralement admis qu'un déficit thermique est à l'origine de cette production limitée et de cette maturation incomplète. Larcher (1995) a décrit les mécanismes de stress liés au froid et Beniston et al. (1996) ont exposé l'action directe et indirecte des basses températures sur les écosystèmes de montagne.

Parallèlement à ces approches écophysiologiques, les biogéographes ont essayé d'établir une corrélation entre l'altitude de la limite supraforestière et les paramètres climatiques. Ainsi, depuis Brockmann-Jerosch (1919), l'altitude de 
l'isotherme de $10^{\circ} \mathrm{C}$ du mois le plus chaud est considérée comme un bon indicateur (Dolukhanov, 1978 ; Arno et Hammerly, 1984 ; Grace, 1987 ; Grant et French, 1990). De là à rechercher un parallélisme entre les fluctuations climatiques et les fluctuations de la limite supraforestière, il n'y a qu'un pas.

Certaines des dépressions générales de la limite supraforestière au cours des 18000 dernières années ont été associées avec des oscillations climatiques majeures (Burga, 1988). Mais à l'échelle régionale, il y a une alternance complexe de phases boisées et de phases non boisées (Borel et al., 1984). La variabilité de la limite supérieure des arbres est fonction des massifs étudiés et l'évaluation de la densité du couvert végétal reste problématique (David, 1993). II est difficile d'interpréter en termes climatiques précis l'évolution observée de la végétation. Selon Lamb (1977 in Cannell et al., 1989) la température estivale de l'air en 6000 BP, c'est-àdire pendant l'optimum thermique de la période atlantique, aurait été de $2^{\circ} \mathrm{C}$ supérieure à celle d'aujourd'hui. En même temps, nous savons que depuis cet optimum thermique du Postglaciaire, il y a eu une régression de 150 à $400 \mathrm{~m}$ de la limite supraforestière en Eurasie et en Amérique du Nord (Holtmeier, 1994) et que dans les Alpes la limite supérieure de la forêt est actuellement de 150 à 300 m (Mathey, in Flahault, 1901 ; Holtmeier, 1987), voire de 400 m (De Beaulieu et al., 1990) inférieure à la limite climatique potentielle. Or, il est difficile de savoir quelles sont les parts relatives de l'intervention humaine et du climat dans cette régression. En définitive, il semble qu'à l'échelle de grands intervalles d'espace et de temps il soit possible d'établir une correspondance entre les fluctuations du climat et celles de la limite supraforestière. Mais à l'échelle régionale, les processus et les relations de cause à effet ne sont pas simples et linéaires. Les communautés végétales, telles qu'elles sont observées aujourd'hui en limite supérieure de forêt, doivent être considérées comme transitoires dans l'espace et dans le temps. Quel peut alors être leur pertinence en tant que bioindicateurs?

En général, les zones de bordure des écosystèmes (écoclines et écotones), surtout celles où des populations végétales poussent près des limites de leur tolérance par rapport à certains facteurs du milieu, sont considérées comme très sensibles à des changements environnementaux. Leur observation permettrait une détection précoce de ces changements (Di Castri et al., 1988 ; Cannell et al., 1989 ; Hansen et al., 1992 ; Holten et Carey, 1992 ; Guisan et al., 1995). Est-ce que la limite supraforestière fait partie des écotones susceptibles d'être rapidement touchés par un réchauffement climatique global ? Son déterminisme essentiellement thermique peut le laisser croire. Cependant, ce déterminisme ne semble pas toujours aboutir à une situation d'équilibre simple entre un facteur (la température) et une formation végétale (la forêt) ou d'un étage de végétation (l'étage subalpin). En effet, cet équilibre repose sur des processus, plus que sur un facteur. Ces processus sont à la fois biologiques (croissance, reproduction, dissémination, compétition...) et physiques (microclimat, érosion, manteau neigeux, pédoclimat...) et se déroulent à des échelles spatio- temporelles multiples. En outre, ils interfèrent avec des processus d'origine anthropique. L'interaction de tous ces phénomène fait en sorte qu'il est difficile de prévoir l'effet d'une variation de la température sur la position précise de la limite supraforestière. Comme les limites supraforestières ne répondent pas aussi automatiquement et promptement à des changements climatiques que la limite des neiges, Holtmeier (1973) dénonce leur assimilation abusive à des isolignes (telle la limite des neiges) et leur utilisation pour caractériser différents types de climat. II préconise des études locales ou régionales pour approfondir nos connaissances sur le phénomène hétérogène qu'est la limite supraforestière.

Les recherches menées depuis quelques années en moyenne Maurienne répondent à cet appel et offrent des perspectives nouvelles par rapport à la réponse de la limite supraforestière à des changements climatiques. Dans le secteur étudié, des conditions pédoclimatiques variées, combinées à une occupation humaine variable dans l'espace et dans le temps, toutes les deux liées à l'hétérogénéité du relief, sont à l'origine d'une diversité importante des écotones supraforestiers. Ainsi, il n'existe pas une limite supraforestière simple, continue et rectiligne, mais un grand nombre de lisières dont les propriétés statiques et dynamiques diffèrent.

\section{L'OBSERVATION DES PHASES RÉCENTES DE COLONISATION LIGNEUSE DANS LES ALPAGES ABANDONNÉS DE MAURIENNE : DÉMARCHE ET RÉSULTATS}

Le caractère complexe, dans le sens de Allen et Starr (1982), de la limite supraforestière, c'est-à-dire dépendant de plusieurs facteurs qui agissent à plusieurs échelles de temps et d'espace, nous a conduit à adopter une démarche pluriscalaire et à mettre en oeuvre une stratification écologique des observations. Nous avons retenu trois niveaux d'observation (fig. 2), qui correspondent à trois façons différentes de percevoir la limite supraforestière. À l'échelle du secteur, elle apparaît comme une frontière entre deux matrices ; à l'échelle des sites, elle constitue une zone écotonale formée d'une mosaïque de phytocénoses ; à l'échelle des placettes, les lisières forestières individuelles deviennent apparentes.

Voici en résumé les principales méthodes d'analyse de la structure et de la dynamique de la végétation aux trois échelles d'observation :

1) À l'échelle du secteur

a. Cartographie diachronique de l'évolution de la végétation ligneuse d'après des cartes topographiques anciennes, échelle d'analyse $1 / 25000$;

b. esquisse géomorphologique des hauts de versants d'après les photographies aériennes.

2) À l'échelle des sites

a. Cartographie phyto-morphologique d'après les photographies aériennes (échelle d'analyse 1/10000) ; 


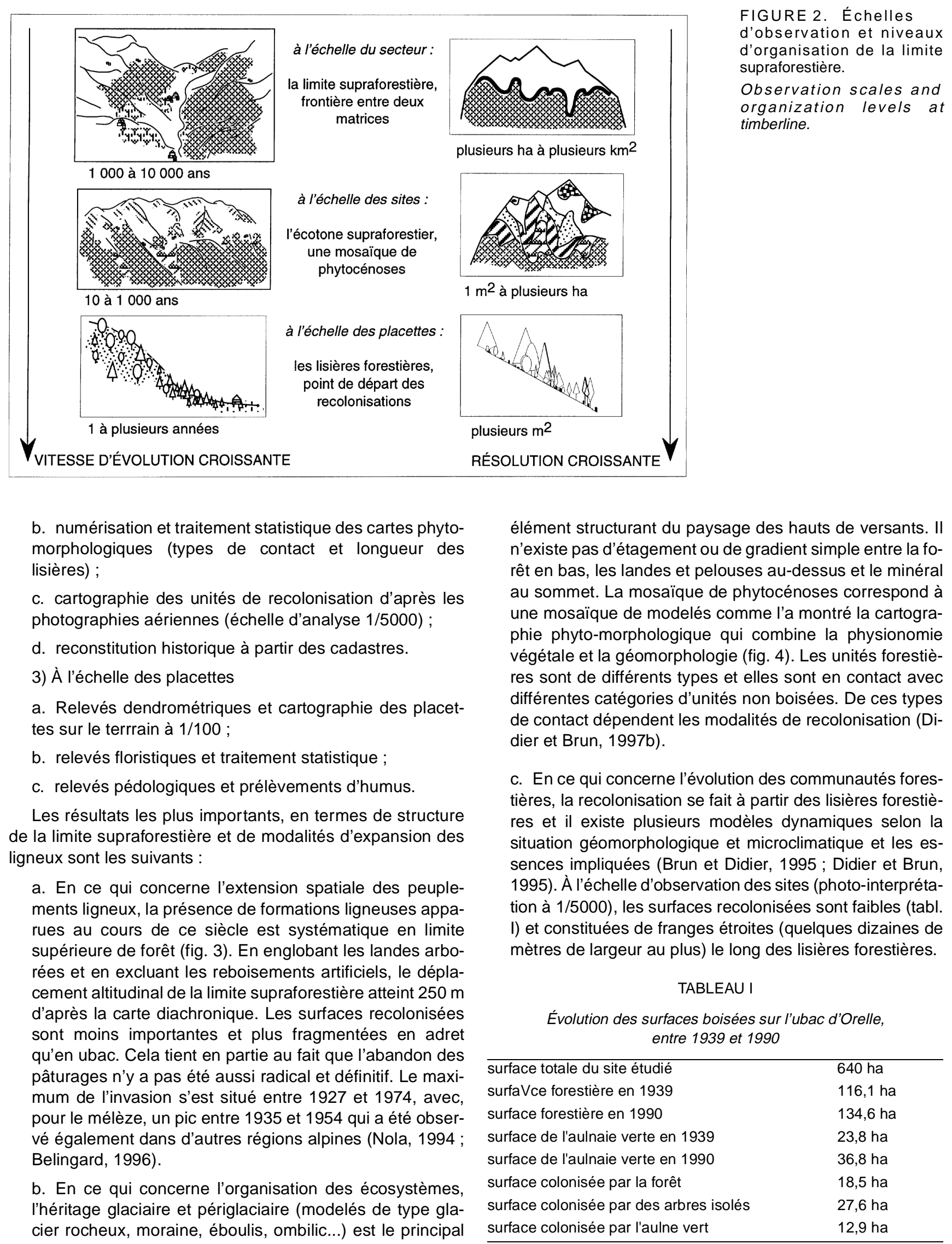


FIGURE 4. Exemple de carte phyto-morphologique de l'ubac d'Orelle.

Example of a phytomorphological map: the northern exposed slope above Orelle.

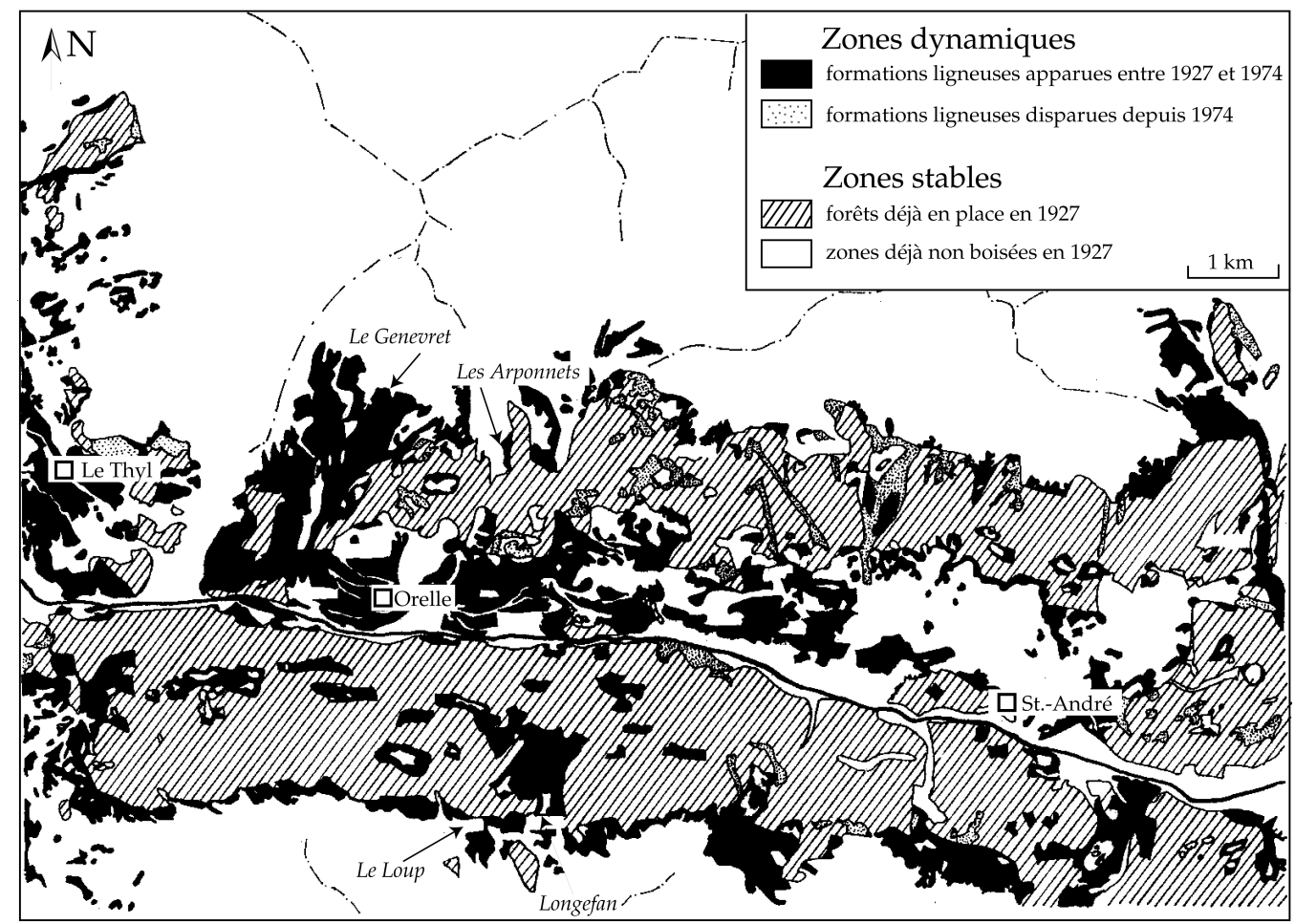

FIGURE 3. Évolution des surfaces forestières entre 1927 et 1974 dans les gorges houillères entre Saint-Michel-de-Maurienne et Modane.

Evolution of forest areas between 1927 and 1974 in the gorges houillères between Saint-Michel-deMaurienne and Modane.
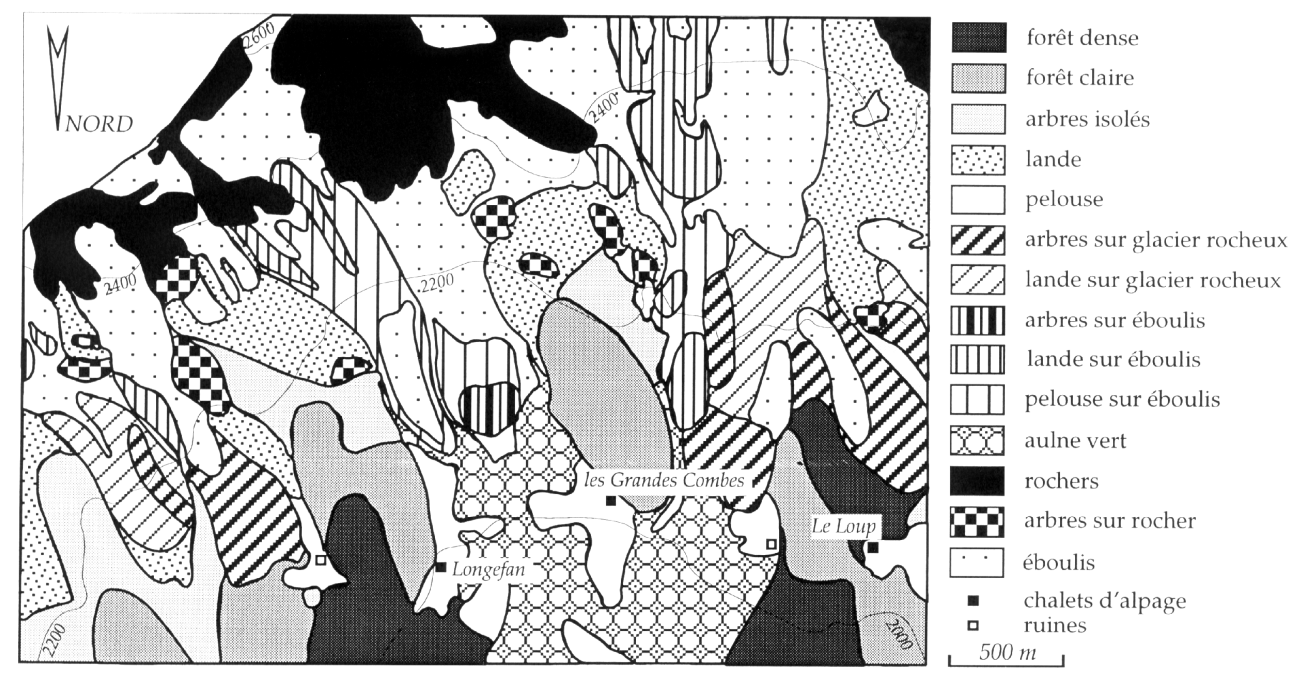

Les trois catégories d'unités de recolonisation, les plus importantes observées par exemple dans un site en ubac, dans la commune d'Orelle, sont les suivantes (fig. 5) :

1. La recolonisation a été la plus rapide et la plus importante aux lisières forestières autour de $2000 \mathrm{~m}$ d'altitude, à côté des chalets abandonnés. Les mélèzes (Larix decidua Miller) y forment des peuplements denses dont la plupart des individus se sont établis entre 1934 et 1954, c'est-à-dire immédiatemment après l'arrêt du pâturage. La régénération dans ces peuplements est formée par le pin cembro (Pinus cembra L.).

2. Un deuxième type de recolonisation se rencontre dans de petits couloirs d'avalanche, au-dessus de la zone des chalets. Ici, le mélèze domine également. L'invasion a démarré au début du siècle, s'est intensifiée entre 1954 et 1974 et se poursuit actuellement. Le pin cembro n'y joue qu'un rôle mineur.

3. A la limite supérieure des arbres, le pin cembro devient dominant. II forme des groupes d'arbres, assez denses, surtout sur les reliefs convexes, comme les rides de moraines et de glaciers rocheux (dissémination par un oiseau, le casse-noix moucheté, qui établit des caches de graines dans les zones peu enneigées). Le recrutement des pins est irrégulier au cours du temps. Les taches de quelques mètres carrés où la densité de semis actuels est très élevée (jusqu'à 130 semis et jeunes arbres sur 100 

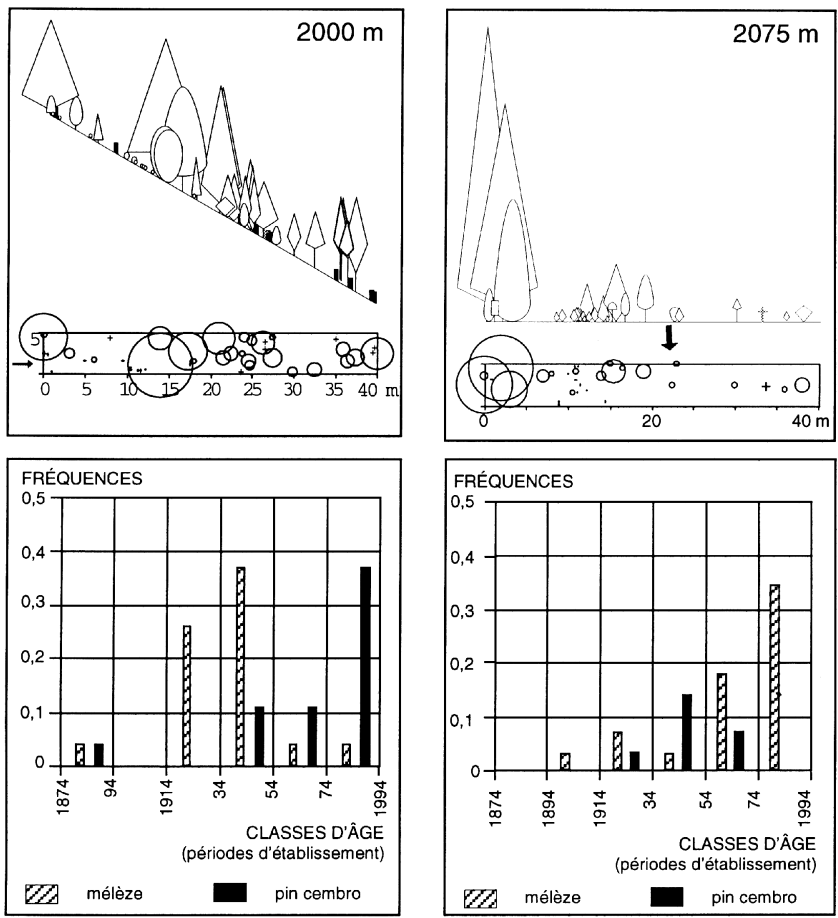

Recolonisation par le mélèze à côté d'un chalet d'alpage

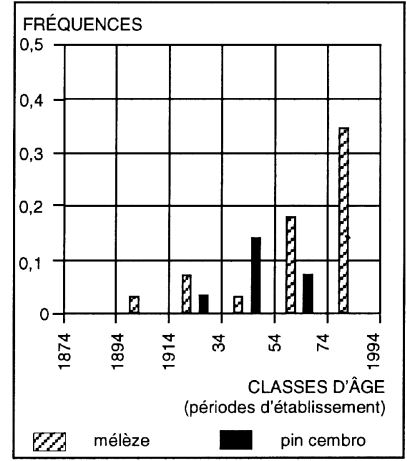

Recolonisation par le mélèze dans un couloir d'avalanche
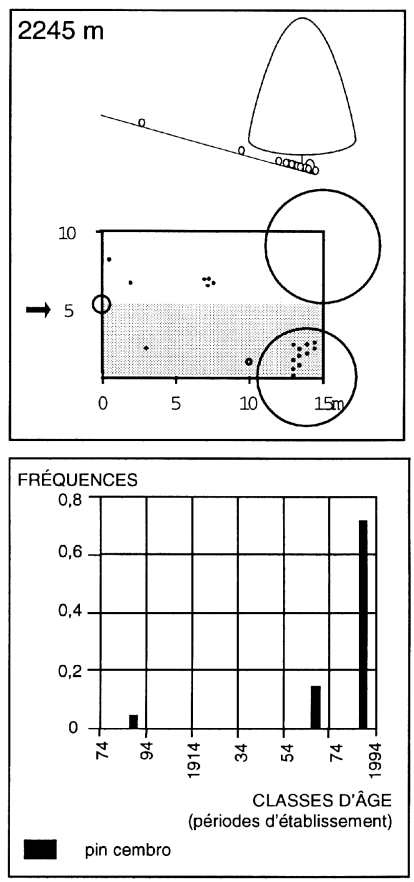

Pins cembros en limite supérieure des arbres, sur un glacier rocheux (en gris de la coupe en plan $=$ zone projetée sur la coupe transversale)

schémas en coupe transversale :
P Pinus cembra
$\triangle$ Larix decidua arbre mort
- souche

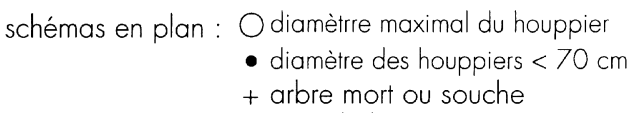

FIGURE 5. Exemples de la structure d'âge sur les placettes de recolonisation en ubac d'Orelle.

Examples of the age structure in recolonization plots. $\mathrm{m}^{2}$ ), sont très fréquentes. Elles se trouvent soit autour de vieux arbres isolés, soit au-dessus de la limite actuelle des arbres. Des semis de pin cembro ont souvent été observés jusque sur les crêtes sommitales du versant, au-dessus de $2600 \mathrm{~m}$ d'altitude.

Cet exemple montre que, selon les conditions locales, les essences impliquées et les vitesses de colonisation diffèrent considérablement, parce que les modalités de dispersion et les conditions de germination varient selon les essences. II est donc important de connaître les mécanismes de régénération des essences avant de les utiliser comme bio-indicateurs.

\section{INTÉRÊTS D'UNE APPROCHE PLURISCALAIRE ET SPATIALISÉE DES ÉCOSYSTĖMES D'ALTITUDE}

Nous avons mis en évidence une grande diversité des successions végétales en cours, en ce qui concerne les espèces impliquées et les vitesses d'invasion et une grande hétérogénéité des conditions de milieu dans l'étage subalpin supérieur (Brun et Didier, 1995 ; Didier et Brun, 1995). Pour pouvoir interpréter cette hétérogénéité, il est nécessaire de « spatialiser » l'information : il faut replacer les lisières étudiées dans leur contexte paysager. Leur place dans la mosaïque phyto-morphologique est déterminante en termes de contraintes géomorphologiques, climatiques et anthropiques, et donc de potentialités d'évolution. Une façon de tenir compte de ce contexte paysager des lisières est de les considérer comme des écotones, c'est-à-dire comme des zones de contact entre deux unités et pas seulement comme contour d'une seule unité. Entre les deux unités en contact, l'une forestière et l'autre pas, il existe un rapport de forces fondé sur le pouvoir de dissémination des arbres d'un côté et sur la réceptivité du substrat de l'autre côté. Cela amène à faire une typologie des écotones, à deux échelles différentes.

1)Les déplacements horizontaux de lisières forestières individuelles à l'échelle des sites et des placettes

La typologie des contacts, basée sur la carte phyto-morphologique, constitue un outil intéressant, permettant une quantification. Ainsi, une grande longueur de lisières entre la forêt claire et les éboulis ou pierriers dans un site donné, indique de faibles potentialités de recolonisation, même en cas de réchauffement du climat, contrairement à des sites où il y a une grande longueur de lisières entre la forêt et la lande arborée (Didier et Brun, 1997b). Il est possible de généraliser cette typologie des contacts en faisant référence à l'origine des écotones. En effet, parmi les lisières forestières, il y en a qui existent indépendamment de l'occupation 
humaine et de la dynamique végétale, parce qu'elles correspondent en fait à une zone de contact entre modelés géomorphologiques dont le substrat se distingue par sa granulométrie, sa stabilité ou son alimentation en eau. On peut ainsi distinguer trois types d'origine qui entraînent des réponses différentes lorsqu'un changement climatique survient.

- Les écotones d'origine géomorphologique (contraintes édaphiques). Lorsqu'il s'agit de modelés hérités non actifs, ces écotones ne sont pas suceptibles de se déplacer sous l'effet d'un changement climatique. Quand il s'agit de modelés encore actifs (solifluxion, éboulisation), un changement climatique peut agir sur les processus d'érosion et provoquer un déplacement de l'écotone. Un changement climatique peut aussi être à l'origine d'une réactivation de processus géomorphologiques, comme c'est actuellement le cas pour certains modelés spécifiques des Pyrénées (Baudière et al., 1985). Les changements de végétation induits sont considérés comme des substitutions et non comme des successions (Baudière et Gauquelin, 1990).

- Les écotones d'origine microclimatique (par exemple, durée du manteau neigeux) sont souvent combinés aux premiers car ils sont liés à la topographie. Ils sont bien sûr susceptibles d'évoluer sous l'influence d'un changement climatique, à travers des substitutions de végétation.

- Les écotones d'origine anthropique coïncident également souvent avec les premiers, car l'homme a utilisé de façon différentielle la mosaïque phyto-morphologique, seIon les qualités fourragères ou pastorales des lieux. La limite des prés correspond souvent à une rupture de pente. En général, le climat n'affecte pas directement ces lisières. Quand elles se déplacent après diminution de la pression anthropique, on parle de succession végétale.

2)Le déplacement altitudinal de la limite supraforestière à l'échelle du secteur

L'approche spatialisée, qui consiste à tenir compte de la nature des unités situées des deux côtés d'un écotone, vient d'être montrée à l'échelle de la mosaïque phyto-morphologique. Or, elle peut aussi s'appliquer à l'échelle du secteur, où la limite supraforestière constitue un écotone entre deux types de systèmes écologiques : en bas, le domaine biostasique de la forêt et, au-dessus, les espaces aforestiers, en grande partie rhexistasiques (c'est-à-dire dont l'évolution est largement dépendante des processus érosifs). Ce n'est pas uniquement une question de couverture végétale, avec dominance de processus biologiques d'un côté et de processus physiques de l'autre, mais aussi de substrat, puisque la limite supraforestière coïncide avec le début de l'espace marqué par l'héritage glaciaire et périglaciaire. Tout comme à l'échelle inférieure, l'espace non boisé qui jouxte la forêt n'est donc pas « neutre».

\section{QUELLES CONSÉQUENCES POUR LES HYPOTHĖSES SUR LES ÉCOSYSTĖMES D'ALTITUDE COMME INDICATEURS DES CHANGEMENTS CLIMATIQUES ?}

Les changements anthropiques, amorcés au siècle dernier dans les zones de montagne, ont provoqué la mise en place de dynamiques végétales qui révèlent le fonctionnement des écosystèmes d'altitude à travers des réactions précoces et des processus à long terme. Il apparaît, en effet, que selon l'échelle d'observation choisie, la dynamique de la limite supraforestière s'exprime par des phénomènes différents. À l'échelle du secteur, le processus en question est la fluctuation des étages bioclimatiques et morphodynamiques. Elle se traduit par le déplacement altitudinal de la limite linéaire entre deux matrices, l'une forestière, l'autre aforestière. À l'échelle des sites, l'évolution de la limite supraforestière correspond au développement des systèmes phytomorphogénétiques constituant la zone écotonale en mosaïque. Elle se traduit par le déplacement de lisières, correspondant à des successions ou des substitutions. À l'échelle des placettes, le processus dynamique correspond aux phénomènes de dissémination, de germination et de compétition. Chacun de ces trois processus est en partie dépendant du climat, mais les paramètres climatiques déterminants ne sont pas les mêmes par rapport à l'échelle spatio-temporelle des évolutions en cours. À l'échelle des sites, par exemple, le climat n'agit pas à travers des paramètres macroclimatiques, mais méso- et microclimatiques, comme la durée et l'épaisseur du manteau neigeux ou le nombre de jours sans gel. Ces paramètres ne dépendent pas directement de la température moyenne annuelle, mais de régimes saisonniers ou des amplitudes journalières (Grant et French, 1990). L'évolution des paramètres méso- et microclimatiques et celle des régimes saisonniers et journaliers peut être disproportionnée ou opposée à l'évolution de la température moyenne. En outre, elle dépend autant des précipitations que des températures.

En ce qui concerne la prévision ou la modélisation des changements du climat, cela implique donc d'abord un choix concernant l'étendue des zones atteintes, les intervalles de temps et les paramètres climatiques à observer, puis le choix des structures écologiques appropriées comme indicateurs.

Souvent, seul le premier des trois processus, c'est-à-dire le déplacement altitudinal de la limite supraforestière, est inclus dans les considérations sur les écosystèmes d'altitude comme indicateurs des changements climatiques. Mais trois problèmes se posent quant à un monitoring des fluctuations de la limite supraforestière à cette échelle.

1. L'altitude de la limite supraforestière est un paramètre qui n'est pas facile à mesurer puisque, même après cartographie, la limite est discontinue et non rectiligne. Son estimation correspond donc à une moyenne.

2. Une extension des forêts ne devient visible dans le paysage et sur des photographies aériennes ou satellitaires qu'après plusieurs décennies. En outre, les change- 
ments structuraux, comme la variation de la densité ou la substitution d'espèces, pourraient être plus importants que les transgressions de limites et sont encore plus difficiles à mesurer.

3. II est difficile de faire la part entre le déterminisme climatique et le déterminisme anthropique dans la remontée des limites forestières. Rappelons que l'amélioration des températures depuis la fin du Petit Âge glaciaire (1450 à 1850 ap. J.-C.), est estimée à 0,5-0,7 ${ }^{\circ} \mathrm{C}$ (Davis, 1986 ; Jones et al., 1986 ; Bücher et Dessens, 1991). Or, la fin du Petit Âge glaciaire coïncide avec la fin de l'économie de subsistance des communautés de montagne et donc le début des mutations socio-économiques aboutissant à l'abandon total des alpages de notre secteur d'étude. À l'échelle des sites et donc pour les déplacements horizontaux de lisières forestières individuelles, il est possible de résoudre en partie le problème de l'interférence de différents facteurs en incluant la dimension spatiale et le contexte géomorphologique.

L'altitude de la limite supraforestière est donc une valeur moyenne et intégratrice, ayant valeur d'indicateur pour des changements se produisant à long terme et sur de grandes surfaces. Elle paraît adaptée à la simulation de ces changements (Grant et French, 1990), mais pas comme indicateur précoce de réels changements en cours. En tout cas et en raison de l'hétérogénéité et de la complexité des milieux supraforestiers, il semble qu'il n'y aura pas de réponse linéaire, sous forme d'une remontée uniforme de l'écotone supraforestier lors d'un réchauffement climatique. C'est aussi l'opinion de Holten et Carey (1992), Holtmeier (1994) et de Weisberg et Baker (1995).

\section{CONCLUSIONS}

La démarche proposée, à la fois pluriscalaire et spatialisée, présente la limite supraforestière sous un nouvel angle et selon plusieurs points de vue. II ne faut pas considérer la limite supraforestière comme un simple contact entre des forêts et des pelouses, à l'image des lisières forestières de plaine. En effet, d'une part, la forêt subalpine est très fragmentée et hétérogène et, d'autre part, les contacts forêtpelouse sont très rares (les pelouses supraforestières ellesmêmes étant très rares dans la zone étudiée). II ne faut pas non plus la considérer seulement du point de vue du couvert végétal. En effet, l'étagement de la végétation se superpose à un étagement morphodynamique et les conditions de substrat sont sensiblement différentes entre l'étage montagnard et l'étage subalpin, sous l'impact des glaciations ayant modelé le haut des versants. D'une façon plus générale, il ne faut pas représenter la forêt subalpine comme un objet d'étude situé au centre d'un environnement dont les facteurs sont tous au même niveau. C'est, en effet, un objet écologique complexe dont les structures à étudier varient selon l'échelle d'observation choisie et qui est en interaction avec des processus qui se déroulent à différentes échelles spatiotemporelles. Ceci est particulièrement vrai pour la relation entre le climat et tous les phénomènes intervenant dans la colonisation d'espaces non boisés (reproduction, dissémina- tion, régénération, dynamique des populations), Ainsi, lors d'un réchauffement global, c'est l'évolution des paramètres micro-et mésoclimatiques locaux qui détermine le déplacement des lisières forestières.

En définitive, l'approche hiérarchique et écosystémique des dynamiques végétales, c'est-à-dire une approche qui comprend à la fois les êtres vivants et leur biotope, à savoir leur territoire avec ses conditions édaphiques et climatiques, pourra seule rendre compte de la complexité des interactions entre le biotique et l'abiotique en montagne.

\section{REMERCIEMENTS}

Nous remercions les lecteurs, MM. Martin Béniston et Réjean Gagnon, pour leurs judicieux commentaires.

\section{RÉFÉRENCES}

Allen, T.F.H., Starr, T.B., 1982. Hierarchy. Perspectives for ecological complexity. The University of Chicago Press, $310 \mathrm{p}$.

Arno, S.F. et Hammerly, R.P., 1984. Timberline. Mountain and arctic forest frontiers. The Mountaineers, Washington, $304 \mathrm{p}$.

Baudière, A., Gauquelin, T. et Serve, L., 1985. La régression des pelouses culminales et les facteurs de la géomorphologie sur les hautes surfaces planes oriento-pyrinéenne. Colloques phyto-sociologiques, 13, Végétation et géomorphologie, Bailleul 1985 : 149-171.

Baudière, A. et Gauquelin, T., 1990. Successions, substitutions, écotones et systèmes phytomorphogénétiques pyrénéens d'altitude. Botanica pirenaico-cantabrica, p. 353-366.

Belingard, C., 1996. Étude dendroécologique de la dynamique de la limite supérieure de la forêt dans les Alpes du Sud, en relation avec les facteurs climatique et anthropique. Thèse d'université, Aix-Marseille III, $103 \mathrm{p}$.

Beniston, M., Fox, D.G., Adbilcary, S., Guisan, A., Holten, J., Innes, J., Maitinn, J., Price, M. et Tessier, L., 1996. The impacts of climate change in mountain regions, p. 191-213. In R.I . Watson, M. Zinyomer, et R.H. Moss, édit., Climate change 1995 : The impact of climate change, adaptation and migration options. Intergovernmental Panel on Climate Change (IPCC), Cambridge University Press.

Borel, J.L., Jorda, M. et Monjuvent, G., 1984. Variations climatiques, morphogenèse et évolution de la végétation post-würmienne dans les Alpes françaises. Ouvrage du Comité international d'Organisation du $25^{\mathrm{e}}$ Congrès international de Géographie, août 1984. Les Alpes, p. 43-53.

Bozon, N. et Grossi, J.L., 1991. Processus d'enfrichement et successions végétales post-culturales en zone de montagne, approche pluridisciplinaire et pluriscalaire. Colloques phytosociologiques, 20, Phytodynamique et biogéographie historique des forêts, Bailleul, p. 175189.

Brockmann-Jerosch, H., 1919. Baumgrenze und Klimacharakter. Berichte der Schweizer Botanischen Gesellschaft, 26, 255 p.

Brun, J.J., Delcros, P. et Grossi, J.L., 1995. Modèle de paysage et gestion de la biodiversité dans les terrains de montagne abandonnés (Alpes Françaises), p. 468-477.In D. Bellan, G. Bonin, et C. Emig, Functionning and dynamics of natural and perturbed ecosystems. Lavoisier, Paris.

Brun, J.J. et Didier, L., 1995. Dynamique des lisières forestières supérieures, L'exemple de la moyenne Maurienne, Alpes françaises du Nord. Rapport final de recherche, Comité EGPN du Ministère de l'Environnement, Cemagref, Grenoble, $35 \mathrm{p}$.

Bücher, A. et Dessens, J., 1991. Secular trend of surface temperature at an elevated observatory in the Pyrénées. Journal of Climate, $4: 859-868$.

Burga, C.A., 1988. Swiss vegetation history during the last 18000 years. New Phytologist, $110: 581-602$.

Cannell, M.G.R., Grace, J. et Booth, A., 1989. Possible impacts of climatic warming on trees and forests in the United Kingdom : A review. Forestry, 62 (4) : 337-364. 
David, F., 1993. Extension tardiglaciaire des pins dans les Alpes du nord. Comptes rendus de l'Académie des Sciences, Paris, série II, 317 : 123129.

Davis, M.B., 1986. Climatic instability, time lags, and community disequilibrium, p. 269-284. In D.J. Diamond et T.J. Case, Community Ecology. Harper \& Row, New York.

De Beaulieu, J.L., Édouard, J.L., Ponel, P., Rolando, C., Tessier, L., Thinon, M. et Thomas, H., 1990. Timberline and human impacts in the French Alps. The state of the art and research programs. P.A.C.T. Revue, $31: 63-80$.

Delcros, P., 1993. Écologie du paysage et dynamique végétale post-culturale. Thèse de doctorat, Université de Grenoble1, Cemagref, Études-gestion des territoires 13, $334 \mathrm{p}$.

Di Castri, F., Hansen, A.J. et Holland, M.M., 1988. A new look at ecotones. Biology International, special issue 17, $163 \mathrm{p}$.

Didier, L. et Brun, J.J., 1995. Swiss stone pine (Pinus cembra L.) and european larch (Larix decidua Miller) dynamics at the upper timberline in the French Alps. Communication orale au7th European Ecological Congress, Eureco '95, 20/08.25/08/1995, Budapest, Hungary.

1997a. Reboisements de protection et sous-utilisation des alpages : impacts écologiques et paysagers. Exemple de la série R.T.M. de la Grollaz, moyenne Maurienne, Alpes françaises du Nord. Ecologia mediterranea, 23 (1/2) :107-119..

1997b. La cartographie phyto-morphologique, un outil d'analyse structurelle et fonctionnelle de la dynamique des lisières forestières subalpines. Colloque en l'honneur des professeurs P. Ozenda et B. Souchier,Végétations et sols de montagnes. Diversité, fonctionnement et évolution, Grenoble, 8-13 juillet 1996. Écologie,29 (1).

Dolukhanov, A.G., 1978. The timberline and the subalpine belt in the Caucasus Mountains, U.S.S.R.. Arctic and Alpine Research, 10 (2) : 409-422.

Flahault, C., 1901. Les limites supérieures de la végétation forestière et les prairies pseudo-alpines en France. Revue des Eaux et Forêts, V (25-27) : 385-401 et 417-439.

Grace, J., 1987. Climatic tolerance and the distribution of plants. New Phytologist, suppl. $106: 113-130$.

Grant, W.E. et French, N.R., 1990. Response of alpine tundra to a changing climate : A hierarchical simulation model. Ecological Modelling, 49 : 205227.

Grossi, J.L., Chenavier, L., Delcros, P. et Brun, J.J., 1995. Effets de la structure d'un paysage en situation de déprise agricole sur la végétation et certains groupes anomaux. Landscape and urban planning, $31: 291-301$.
Guisan, A., Holten, J.I., Spichiger, R. et Tessier, L. (édit.), 1995. Potential ecological impacts of climate change in the Alps and Fennoscandian mountains. Ed. Conserv. Jardin botanique de Genève, hors-série 8, $194 \mathrm{p}$.

Hansen, A.J., Risser, P.G. et Di Castri, F., 1992. Epilogue : Biodiversity and ecological flows across ecotones, p.423-438. In A.J. Hansen et F. Di Castri, édit., Landscape boundaries. Consequences for biotic diversity and ecological flows. Springer-Verlag, New York.

Holten, J.I. et Carey, P.D., 1992. Responses of climate change on natural terrestrial ecosystems in Norway. NINA forskningsrapport 29, 59 p.

Holtmeier, F.K., 1973. Geoecological aspects of timberlines in northern and central Europe. Arctic an Alpine Research, 5 (3) : A45-A54.

1987. Human impacts on high altitude forests and upper timberline with reference to middle latitudes, p. 9-20.. In T. Fudjimori et M. Kimura,édit., Human impacts and management of mountain forests. Forestry and Forest Products Research Institute, Ibaraki.

1989. Ökologie und Geographie der oberen Waldgrenze. Rintelner Symposium I, 17.-18.3. 1989. Berichte der Reinhold Tüxen-Gesellschaft, Göttingen, $1: 15-45$.

1994. How does the timberline respond to changing climate ? In M Beniston, édit., Mountain environments in changing climates. Proceedings of an international conference, 11-16 oct. 1992, Davos, Switzerland.Routledge, London.

Jones, P.D., Wigley, T.M. et Wright, P.B., 1986. Global temperature variations 1861-1984. Nature, $322: 430-434$

Lamb, H.H., 1977. Climate: Present, past and future, 2. Climatic history and the future. Methuen, London.

Larcher, W., 1995. Physiological plant ecology. Ecophysiology and stress physiology of functionnal groups. Springer, $506 \mathrm{p}$.

Mathey, A., 1900. Le pâturage en forêt.

Nola, P., 1994.- A dendroecological study of larch at timberline in the Central Italian Alps. Dendrochronologia, 12 : 77-91

Slayter, R.O. et Noble, I.R., 1992. Dynamics of montane treelines, p. 346- 359. In A.J. Hanse et F. Di Castri, édit., Landscape boundaries. Consequences for biotic diversity and ecological flows. Springer-Verlag, New York.

Tranquillini, W., 1979. Physiological ecology of the alpine timberline. Ecological Studies 31, Springer Verlag, $137 \mathrm{p}$.

Troll, C., 1973. The upper timberlines in different climatic zones. Arctic an Alpine Research, 5 (3) : A3-A18.

Weisberg, P.J. et Baker, W.L., 1995. Spatial variation in tree seedling and krummholz growth in the forest-tundra ecotone of Rocky Mountain National Park, Colorado, U.S.A. Arctic and Alpine Research, 27 (2) : 116-129. 